\title{
Comparison of spatially and spectrally resolved solar data with numerical simulations
}

\author{
G. Cauzzi ${ }^{1}$, A. Asensio Ramos ${ }^{2}$, K. P. Reardon ${ }^{1}$ and K. Janssen ${ }^{1}$ \\ ${ }^{1}$ INAF - Osservatorio Astrofisico di Arcetri, I-50125 Firenze, Italy \\ email: [gcauzzi, kreardon, kjanssen]@arcetri.astro.it \\ ${ }^{2}$ Instituto de Astrofisica de Canarias, E-38205 La Laguna (Tenerife), Spain \\ email: aasensio@iac.es
}

\begin{abstract}
We present a detailed comparison between high resolution observations of a quiet solar region and simulated spectra in several 3-D snapshots of a realistic radiation-hydrodynamical simulation of the solar atmosphere. We find excellent agreement between the two data sets, thus providing strong support to the realism of the simulations, and confirming the high quality of the instrumentation. We propose that spatially resolved spectral data can be employed as a complementary tool to more classic analysis for investigating the hotly debated topic of solar abundances.
\end{abstract}

Keywords. Sun: photosphere, Sun: abundances, line: profiles, instrumentation: high angular resolution, convection

\section{Introduction}

Time-dependent, parameter-free, 3-D hydrodynamical simulations of solar surface convection, such as those pioneered by Stein \& Nordlund (1989), have been able to reproduce many observed properties of the non-magnetic solar photosphere (see e.g. the review by Stein \& Nordlund 2000). A stringent test of the reliability of such simulations for the atmospheric layers above the solar surface has been provided by the excellent agreement between predicted and observed averaged shapes, shifts and asymmetries for weak $\mathrm{Fe}$ lines in the quiet Sun (Asplund et al. 2000b). These results have lent support to the subsequent use of simulations for precise determination of both solar and stellar surface abundances, leading in some cases to significant differences with the values obtained by classical 1-D analysis (see, e.g., the contribution by Asplund in these proceedings).

Averaged spectral line profiles have the clear advantage of bypassing difficulties related to atmospheric seeing and instrumental resolutions. However, the comparison of simulation results with spatially resolved spectral observations will eventually become necessary for checking the precise structure of the upper solar photosphere and its temporal evolution (for example regarding the presence of high frequency waves, see de Wijn et al. 2005), the effects of small scale magnetic features (Janssen et al. 2003; Khomenko et al. 2005), or the line formation process (Kiselman \& Asplund 2001). Further, the use of spatially resolved data, combined with a reliable knowledge of instrumental performances, might prove helpful in clarifying some issues still debated about the simulations, such as the derived granular contrast, much higher than what observed, or even the reliability of the abundances determination itself (Keller 2006; Ayres et al. 2006).

In this paper we present a first attempt at this task, by comparing spectral lines synthetized in simulations of the quiet solar photosphere with highly resolved data obtained with the Interferometric BIdimensional Spectrometer (IBIS, Cavallini 2006) installed at 

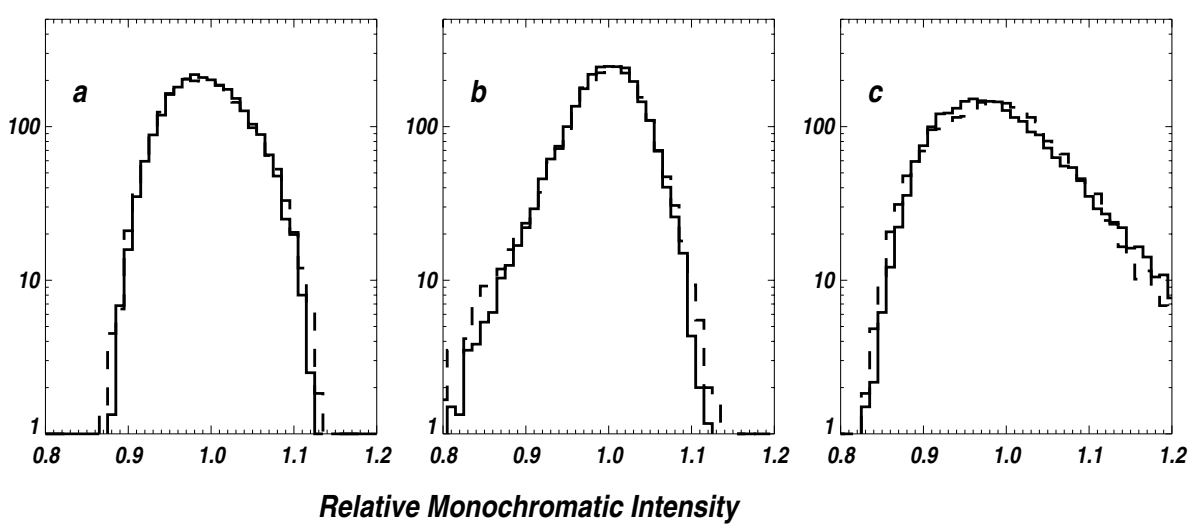

Figure 1. Brightness distributions for images acquired in the continuum $(a),-9 \mathrm{pm}$ from line core $(b)$, and at line core $(c)$. Solid represents simulated data (after degradation); dashed represents observed data. The x-axis is normalized to the monochromatic average value; the $\mathrm{y}$-axis scale is such that the integral of the distribution is equal to the number of pixels in a snapshot.

the Dunn Solar Telescope of NSO (USA). This double interferometric system combines the advantages of a full spectroscopic analysis with the high spatial resolution, high temporal cadence and large field of view (FOV) typical of filter instruments.

\section{Data analysis}

Observations: IBIS is used with high-order adaptive optics (Rimmele 2004) and provides narrow-band images in the range 580-860 $\mathrm{nm}(\mathrm{FWHM}=2-4.5 \mathrm{pm})$. The spectral passband is sequentially stepped through multiple wavelengths in a number of selected lines, providing full spectral information over a circular FOV $80^{\prime \prime}$ in diameter. The data utilized here were obtained in the photospheric, non-magnetic Fe I $709.0 \mathrm{~nm}$ line in a quiet region at disk center on June 2, 2004, for about one hour of good seeing conditions (Janssen \& Cauzzi 2006). The line was sampled in about $4 \mathrm{~s}$ (16 spectral positions with an average step of $3 \mathrm{pm}$ ) and with an image scale of $0.17^{\prime \prime}$ per pixel. For comparison with simulations (see below), we utilize a subset of $150 \times 100$ pixels $\left(25^{\prime \prime} \times 17^{\prime \prime}\right)$ from a series with very good seeing, carefully chosen to avoid any magnetic network area. We have verified that similar results are obtained irregardly of the chosen time step.

Synthetic data: Spectral synthesis of the Fe I $709.0 \mathrm{~nm}$ line was performed in LTE, for six snapshots of the recent 3-D radiation-hydrodynamical simulation of Asplund et al. (2000a). The volume covered by the grid is $6 \times 6 \times 3.8 \mathrm{Mm}$, with a horizontal step size of 30 $\mathrm{km}$. After the synthesis, the data has been rebinned to a step size of $120 \mathrm{~km}$, comparable to the sampling of our observations, thus providing six independent solar scenes, each $50 \times 50$ pixels wide. The iron abundance was set to the classical value of 7.67 , and the oscillator strength of the line at $\log g f=-1.21$ (Nave et al. 1994).

Instrumental effects: In order to perform a meaningful comparison, we further degraded the synthetic data to the actual spatial and spectral resolution of the observations. For the spatial degradation, we followed the customary approach (Nordlund 1984; Collados \& Vázquez 1987), accounting for the PSF of both the telescope (assumed of 60 $\mathrm{cm}$ diameter - this provides a diffraction limit close to that set by the image scale in the observations) and the terrestrial atmosphere. The relevant parameters were set to match simultaneously the average contrast and spatial power spectra of the synthetic 
continuum images with the observed values (see discussion in Keller 2006). All the other results derive solely from this choice of parameters.

The spectral degradation was performed by convolving the synthetic profiles with the IBIS transmission which is known to a high level of precision (Reardon 2006, private communication). Finally, for each snapshot a subset of 16 'narrowband images' corresponding to the wavelengths of the observations has been extracted from the degraded synthetic data, in order to completely mimic the IBIS data. All spectral line parameters are then derived in the same exact way for both data sets.

\section{Results}

Fig. 1 shows the comparison between the observed and synthetic brightness distribution for three wavelengths within the line. The similarity is quite remarkable, and we remind that only the average contrast in the continuum has been used to tie the two sets of data. The slightly asymmetric distribution seen in the continuum (panel $a$ ) is typical of the granulation pattern after spatial smearing (Stein \& Nordlund 2000). The distribution evolves into one much skewed towards low values when sampling in the blue wing of the line $(-9 \mathrm{pm}$, panel $b)$. This betrays the convective upflow of granules, that shifts the spectral line towards the blue with respect to average: when observing at a fixed wavelength in the blue wing, the granules will appear darker because one is actually sampling wavelengths closer to the core of their spectral profiles. The asymmetry subsides further in the wing and eventually reverses already from the line core wavelength (panel $c$ ) when granules are brighter than average (see also Janssen \& Cauzzi 2006).

Such a pattern is more clearly revealed from the monochromatic images themselves, shown in Fig. 2 for the degraded simulations (top row) and the observations (bottom row) at various wavelengths within the line. This figure shows an impressive amount of morphological agreement, and the important cross-talk between convective velocities and intensities just described appears obvious.

Further evidence that the simulations provide results very close to the actual observations is given in Fig. 3, where the line-of-sight (LOS) velocity, calculated as the Doppler shift of the line minimum intensity, is compared. We remind that, even if the exact hydrodynamical velocities are available for the simulated spectra, we have derived this quantity in a fully consistent way for both synthetic and observed data. Panel $a$ provides the distribution of the LOS velocities (negative values correspond to upflows), and we
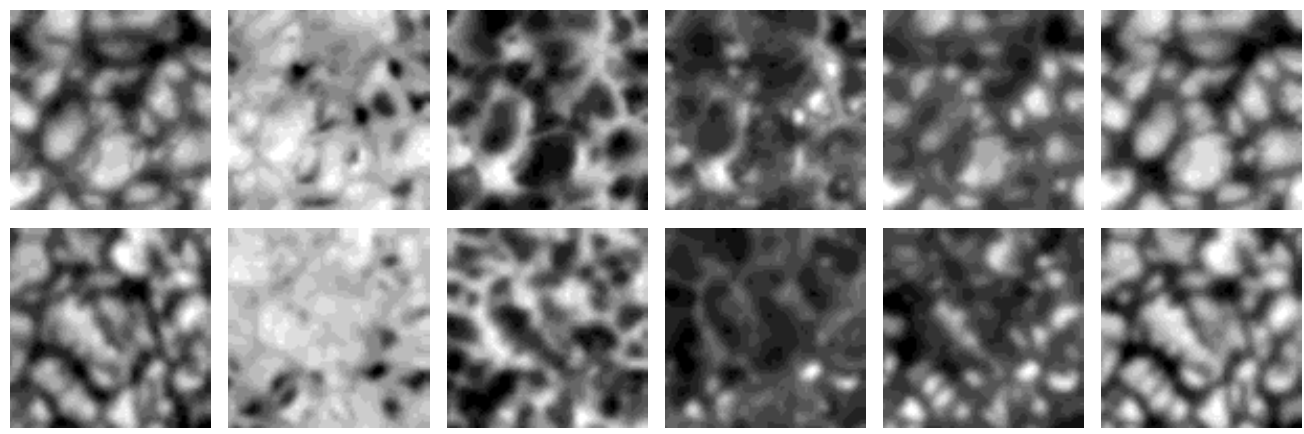

Figure 2. Monochromatic images at several wavelengths within the FeI 709.0 nm line. From left to right: continuum, $-9,-3,0,+3,+9 \mathrm{pm}$ from nominal line core. Top row: synthetic data, after smearing with instrumental and telescope profile; bottom row: IBIS observations. FOV is $6 \times 6 \mathrm{Mm}$. Intensity is scaled at each wavelength for best display. 

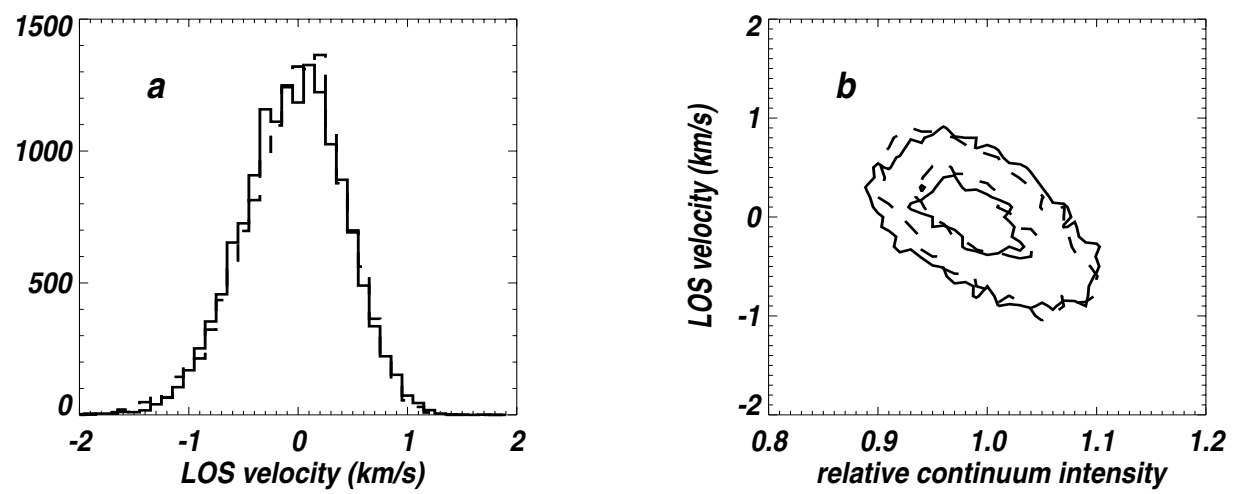

Figure 3. Panel $a$ : Distribution of LOS velocity for simulated (solid) and observed (dashed) data. Negative values indicate upflows. Panel $b$ : scatterplot of LOS velocity vs. continuum intensity, for simulated (solid) and observed (dashed) data. Contours are set at $40 \%$ and $80 \%$ of total number of pixels in the sample.

can notice the same asymmetry found in the brightenss distribution in the continuum (Fig. 1 a), due to the different areas occupied by granules and intergranules. Since the LOS velocity of the average line profile has been set to zero, the distribution peaks at slightly positive values. Panel $b$ provides the scatterplot of the LOS velocities vs. the continuum intensity, essentially summarizing the same physics.

A number of other line quantities (the value of intensity contrast with wavelength, the correlation between intensities in the continuum and within the line, etc.) have been verified and again compare very satisfactorily between the simulation results and the observed data (Asensio Ramos et al. 2006, in preparation). All of these quantities basically describe the relative behavior of the lines over the granulation pattern, i.e. the interplay between intensity and vertical velocity in the layers sampled by the spectral line. The almost perfect agreement with observations provides a strong support to the validity of the simulated atmosphere.

Finally, let's get back to the actual spectral profiles, that encode the same physics as above plus an additional parameter, namely the elemental abundance. In Fig. $4 a$ we show the spatially and temporally averaged line profile of Fe I $709.0 \mathrm{~nm}$ for the original simulations (solid), the simulations after convolving with the IBIS spectral transmission profile (dotted) and the actual observations (dashed). We can see that the simulated profiles are a few percent stronger than observed. An obvious check to perform is the comparison of the observations with the atlas (Fig. 4b): the agreement is excellent, with differences amounting to less than $1 \%$. We hence believe that the abundance we used is slightly too high, in agreement with earlier determinations (M. Asplund, private communication). We are currently testing which abundance will provide the best fit between observed and synthetized line profiles, while preserving the results discussed before.

\section{Conclusions}

We have compared a set of high quality, spatially and spectrally resolved data of the quiet solar photosphere obtained in the Fe I $709.0 \mathrm{~nm}$ line, with the results of line synthesis for several snapshots of realistic granulation simulations. After careful consideration of the instrumental effects, both on the spatial and spectral resolution, we find an excellent agreement between observations and simulations, for many parameters that describe the 

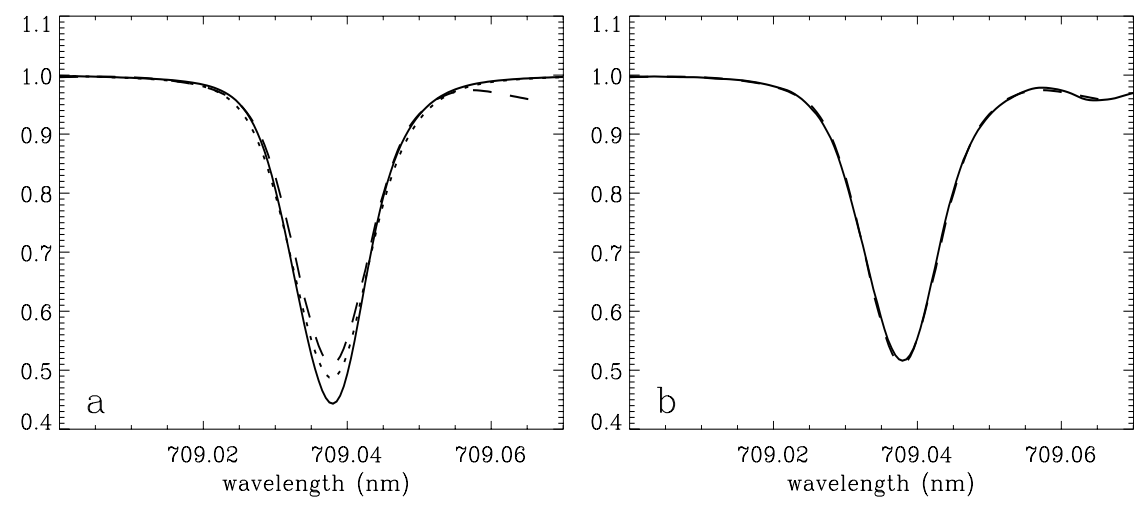

Figure 4. Panel $a$ : Temporally and spatially averaged spectral profiles for simulations (solid), simulations after convolution with IBIS spectral transmission (dotted) and observed data (dashed). Panel $b$ : Same as $a$, but solid now is for FTS atlas, convolved with IBIS transmission.

relative behavior of the lines over the granulation pattern. These results clearly support the use of numerical simulations as synthetic atmospheres in order to retrieve abundances. In this respect, from our data we derive that the classical iron abundance $([\mathrm{Fe}]=7.67)$ is slightly too high to reproduce the observations.

In general, our results provide a strong support to the realism of the simulations, as well as a strong validation of the performances of the IBIS instrument. We remark that the Fe I line employed in this work is probably one of the best choices for this type of comparison: line transition parameters are well known; the line is safely formed within the range of validity of the numerical simulations (Cauzzi et al. 2005); the line is magnetically insensitive. The latter property in particular assures us that any modification of the spectral profile is to be ascribed to thermodynamical and kinematic properties only, rather than to splitting due to small, strong magnetic concentrations thought to densely populate the intergranular areas even in quiet Sun (Domínguez Cerdeña et al. 2003, Trujillo Bueno in these proceedings). It will be of interest to investigate if, and how, these magnetic structures affect the average profiles of lines with different magnetic sensitivity and thus bias the abundance determination (Keller 2006). In this respect, we plan to perform the same kind of analysis presented in this paper by means of simultaneous observations with IBIS in both the Fe I $709.0 \mathrm{~nm}$ and the magnetic Fe I $617.3 \mathrm{~nm}$ line $\left(g_{L}=2.5\right)$.

\section{Acknowledgements}

We are very grateful to M. Asplund for providing the simulation snapshots as well as constructive comments. IBIS was built with contributions from INAF/Arcetri Observatory, the University of Florence, the University of Rome Tor Vergata, and MIUR. NSO is operated by the Association of Universities for Research in Astronomy, Inc. (AURA), under cooperative agreement with the National Science Foundation. This research was partially funded through the European Solar Magnetism Network (ESMN, contract HPRN-CT-2002-00313) and the Italian Ministry for University and Research grant PRIN-MIUR 2004. 


\section{References}

Asplund, M., Ludwig, H.-G., Nordlund, Å. \& Stein, R.F. 2000a, A\&A 359, 669

Asplund, M., Nordlund, Å., Trampedach, R., Allende Prieto, C. \& Stein, R.F. 2000b, A\&̛A 359, 729

Ayres, T.R., Plymate, C. \& Keller, C.U. 2006, ApJS 165, 618

Cauzzi, G., Asensio Ramos, A., Reardon, K. \& Janssen, K. 2005, in ESA SP-600: The Dynamic Sun: Challenges for Theory and Observations

Cavallini, F. 2006, Solar Phys. 236, 415

Collados, M. \& Vázquez, M. 1987, A\&A 180, 223

de Wijn, A.G., Rutten, R.J. \& Tarbell, T.D. 2005, A\&\&A 430, 1119

Domínguez Cerdeña, I., Kneer, F. \& Sánchez Almeida, J. 2003, ApJL 582, L55

Janssen, K. \& Cauzzi, G. 2006, A\&A 450, 365

Janssen, K., Vögler, A. \& Kneer, F. 2003, A\&广A 409, 1127

Keller, C.U. 2006, in ASP Conf. Ser. 354, ed. H. Uitenbroek, J. Leibacher \& R. Stein, 3

Khomenko, E.V., Shelyag, S., Solanki, S.K. \& Vögler, A. 2005, A $\& A 442,1059$

Kiselman, D. \& Asplund, M. 2001, in ASP Conf. Ser. 223, 684

Nave, G., Johansson, S., Learner, R. C.M., Thorne, A.P. \& Brault, J.W. 1994, ApJS 94, 221

Nordlund, A. 1984, in Small-Scale Dynamical Processes in Quiet Stellar Atmospheres, 174

Rimmele, T.R. 2004, in Advancements in Adaptive Optics. Proceedings of the SPIE, Volume 5490, ed. D. Bonaccini Calia, B.L. Ellerbroek \& R. Ragazzoni, 34-46

Stein, R.F. \& Nordlund, Å. 1989, ApJ 342, L95

Stein, R.F. \& Nordlund, Å. 2000, Solar Phys. 192, 191

\section{Discussion}

ZAHN: In the abstract you wrote, you stated that the simulated vertical velocities were larger than the observed ones. Here in your talk they seemed to match very well.

CAUzzi: Indeed, a preliminary version of this work showed rather large differences between the velocity distribution of the data vs. the simulations. However, after taking into account more carefully the effect of photometric noise (that reduces sensibly the measured amplitudes), as well as adding more snapshots to the sample, the differences have almost disappeared.

Martinez Pillet: A recommendation would be to analyze intergranular lanes and granules separately to see if both provide the same abundance.

CAuzzi: Thank you for your suggestion. It could represent an alternative way to investigate the presence of small scale, strong magnetic structures in the intergranular quiet Sun.

TOOMRE: In your observations, how do you correct seeing variations over the course of four seconds during which your interferometer is scanning through the spectral line?

CAUzzI: Every narrowband frame is acquired strictly simultaneously with a broadband image $(\mathrm{FWHM} \sim 10 \mathrm{~nm}$ ) at around $700 \mathrm{~nm}$. By comparing the latter over the course of the sequential line scanning, and assuming that in those few seconds the solar scene remains unaltered, one can recover point-to-point distortions due to residual seeing effects after the adaptive optics (AO) corrections. The narrowband images are then re-morphed according to these distortions. We haven't applied any image restoration technique (e.g. speckle or blind deconvolution), because it is yet unclear if and how much they affect the photometry. 